\title{
Affective Response and Enjoyment to Acute High- Intensity Training with Different Intervals in Young and Obese Females
}

\author{
Shuoqi Li', Jiaolei Zhang ${ }^{2}$, Cong Wang ${ }^{1}$, Xiangui Zhu' ${ }^{1}$, Hongru Shi ${ }^{1 *}$ \\ 'Hebei Normal University, Shijiazhuang/Hebei, China \\ ${ }^{2}$ Hebei Province Geriatric Hospital, Shijiazhuang/Hebei, China
}

Received: November 10, 2018
Accepted: February 01, 2019
Published online: April 30, 2019
Keywords:
Enjoyment
Feeling
Frequency of Interval
High-Intensity Interval Training

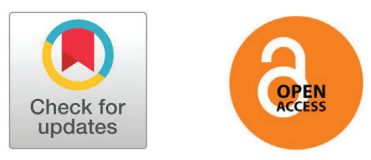

\section{ABSTRACT}

OBJECTIVES To compare the affective responses and enjoyments to acute high-intensity training (HIIT) with short or long intervals in young and obese females.

METHODS Participants completed two bouts of HIIT which were separated over 48 hours respectively. The exercise protocols were cycling on an ergometer at $90 \% \mathrm{VO}_{2 \text { peak }}$ intensity for 4 minutes with 3 minutes recovery(Low-frequency interval, Group L) or 40 seconds cycling with 30 seconds recovery (High-frequency interval, Group H) until $200 \mathrm{KJ}$ mechanical work was completed. At the time of $0 \%, 25 \%, 50 \%, 75 \%$ and $100 \%$ work was completed, the feeling scale (FS), rating of perceived exertion (RPE) were recorded, and the blood lactic acids (LA) were measured when the $50 \%$ and $100 \%$ work was completed. The physical activity enjoyment scale (PAES) was recorded 5 minutes after the exercise.

RESULTS Before the exercise, there were no significant differences between the group $\mathrm{H}$ and $\mathrm{L}$ in terms of the HR, RPE, FS and LA. When $25 \%, 50 \%, 75 \%$ and $100 \%$ of work was completed, the HR and the RPE in group $\mathrm{H}$ were all lower than the corresponding time point in group $\mathrm{L}$. The LA in group $\mathrm{H}$ was lower than the corresponding values in group $L$ when $50 \%$ and $100 \%$ work was completed. At the time of $75 \%$ and $100 \%$ work was completed, the FS in group $\mathrm{H}$ was higher than group $\mathrm{L}$, although there were no significant differences at the time of $25 \%$ and $50 \%$ work done between the two groups. By the end of exercise, the PAES score in group $H$ was higher than group $L$.

CONCLUSIONS Increasing the frequency of interval could increase the positive feeling during HIIT and improve the physical activity enjoyment.

(c) The Asian Society of Kinesiology and the Korean Academy of Kinesiology

\section{前言}

传统的运动减肥形式多采取中等强度持续训练 (moderate intensity continuous training, MICT), 但运动形式单一, 过程枯燥, 时间较长, 导致参与者因 “ 缺乏时间” 和 “缺乏乐趣” 等原因而难以坚持 [1]。近年 来，有研究证据表明高强度间歇训练（High-intensity Interval Training, HIIT) 对减肥更具时效性, 且运

*Correspondence: Shi Hongru, College of Physical Education, Hebei Normal University, Shijiazhuang, Hebei Province; Email: shr2035846@163.com
动愉悦性更高 $[2,3]$ 。其原因可能是由于HIIT较MICT穿 插了更多的间歇, 多变的运动形式提高了运动的愉悦性 [4]，而运动愉悦性的提高能使人们更好地坚持运动 [5]。

HIIT的减肥运动方案并不统一，仍然在不断地调整与 优化 [6]。以往的研究多关注运动强度和运动量的变化, 而不同间歇频率所产生的影响鲜有研究。Tucker WJ等人 指出，在相同运动强度及时间下高频间歇（16×1min） 比低频间歇 $(4 \times 4 \mathrm{~min})$ 运动时所表现的心率更低, 乳 酸堆积更少，而血乳酸堆积是剧烈运动期间产生负面情 感的决定因素之一 $[5,7]$ 。 
那么相同强度及运动量下提高HIIT方案的间歇频率是 否可以通过减少血乳酸来提高运动愉悦性呢? 本研究重 点从运动愉悦性的角度, 比较相同强度及运动量下不同 间歇频率的HIIT方案对肥胖女青年运动中的感觉以及运 动后喜爱程度的影响。

\section{研究方法}

\section{研究对象}

招募18-22周岁青年女性，入选标准如下：1）身体质 量指数 (Body Mass Index, BMI) $\geqslant 24.0 \mathrm{~kg} / \mathrm{m}^{2} ; 2$ ) 体 脂率 $($ FAT\% $)>30 \% ； 3 ）$ 体重近3个月无变化 $( \pm 2 \mathrm{~kg})$ ；4）除每周两次体育课, 无系统的运动锻炼及其它规 律的运动习惯; 5）无代谢, 激素, 骨骼或心血管病史, 且近期未接受药物治疗。经篮选合格受试者 7 名, 基本情 况如表Table 1所示。

\section{实验设计}

受试者首先进行最大摄氧量 $\left(\mathrm{VO}_{2 \text { peak }}\right)$ 测试, 根据结果 确定每个受试者 $90 \% \mathrm{VO}_{2 \text { peak }}$ 运动强度所对应的踏车功率。随 后随机完成两次不同间歇频率的HITT测试：1） $90 \% \mathrm{VO}_{2 \text { peak }}$ 强度低频间歇运动（L组）；2） $90 \% \mathrm{VO}_{2 \mathrm{peak}}$ 强度高频间歇运 动 (H组)。每次测试至少间隔48 小时, 对比两种测试的 运动感觉, 运动喜爱程度, 主观体力感觉及血乳酸情况。

所有测试均在 $13: 00-16: 00$ 间进行, 午餐统一（能量 约 $728 \mathrm{KJ}$, 蛋白质 $7.4 \mathrm{~g}$, 脂肪 $7.2 \mathrm{~g}$, 碳水化合物 $21 \mathrm{~g}$ ）。

\section{运动方案}

首先进行 $5 \mathrm{~min}$ 低强度热身活动, 然后进行正式运动测 试。L组以 $90 \% \mathrm{VO}_{2 \text { peak }}$ 强度在功率自行车上（Monark 839E） 进行 4 min运动（转速保持在60转/分钟）然后休息 $3 \mathrm{~min}$, 再进行下一组 $4 \mathrm{~min}$ 运动, 直至完成200KJ机械功。H组以 同样强度运动 $40 \mathrm{~s}$ 然后休息 $30 \mathrm{~s}$, 直至克服 $200 \mathrm{KJ}$ 机械功。

\section{数据采集}

由于各受试者运动总组数不同, 为保证在相同运动量
下进行对比, 参照先前研究中使用的类似方法 [8], 首先 选取运动前的基线（0\%时）情况进行数据采集，随后评估 完成 $25 \%, 50 \%, 75 \%$ 及 $100 \%$ 运动量时各数据（具体数据采 集点见指标测试部分）。

\section{测试指标}

\section{运动感觉量表}

运动感觉量表（Feeling Scale, FS) 通常用来测量在 没有经过深入思考的情况下引发的本能情感反应。在最近 的HIIT研究中, 经常用FS来测量情感反应 $[9,10]$ 。FS是单 项 11 点双极评定量表, 最大值为 5 , 最小值为 -5 , 表示情感 效价 “积极” 到 “消极”。受试者提前熟悉量表内容, 要 求在运动前 $(0 \%)$ 对其基线情感反应进行评分, 然后分 别在完成 $25 \% ， 50 \% ， 75 \%$ 以及 $100 \%$ 运动量即刻再次评分。

\section{运动喜爱程度量表}

运动喜爱程度量表 (Physical Activity Enjoyment Scale，PAES）采用Kendzierski1991版 ${ }^{[11]}$ 。每次运动测 试结束后静息5分钟, 然后填写量表。

\section{主观体力感觉量表及心率}

通过Brog 6-20的RPE等级量表及心率带（Polar，芬 兰），评估其运动前基线（0\%）以及运动时（总组数的 $25 \% ， 50 \% ， 75 \%$ 以及 $100 \%$ ）的主观体力感觉及心率情况。

\section{血乳酸}

分别在 $0 \%$ 、 $50 \%$ 及 $100 \%$ 三个时间点采指尖血用于测试 血乳酸（ $h / p / \operatorname{cosmos}$ sirius，德国）。

\section{$\mathrm{VO}_{2 \text { peak }}$ 测试及运动强度确定}

均采用Monark839E功率车进行分级递增负荷的试验 （初始负荷为50w，每2min递增20W）。要求受试者需以 $60 \pm 5 \mathrm{r} / \mathrm{min}$ 的速度进行蹬踏, 摄氧量数据通过Quark ${ }^{-}$ PFT- Ergo气体代谢分析仪收集。每级负荷开始前 $10 \mathrm{~s}$ 纪录心率及RPE。判定达到峰值摄氧量的标志：1）呼吸商

Table 1. List of basic information of research subjects

\begin{tabular}{ccccc}
\hline Age (year) & Height $(\mathbf{c m})$ & Body weight $(\mathbf{k g})$ & BMI (kg/m $\left.{ }^{2}\right)$ & VAT\% \\
\hline $19.86 \pm 0.69$ & $159.21 \pm 6.07$ & $67.36 \pm 7.14$ & $26.61 \pm 3.09$ & $32.83 \pm 2.98$ \\
\hline
\end{tabular}


１.15。2）受试者力竭, 经鼓励不能维持预定转速。3) 随着运动强度的增加摄氧量水平达到平台期。通过 $0_{2 \text { peak }}$ 的 线性回归方程计算得出 $90 \% 0_{2 \text { peak }}$ 强度时的功率，同时计算 出运动需要的总运动时间。

\section{统计学分析}

使用Shapiro-Wilk normality test对各项指标数据进 行正态分布判断。采用双因素重复测量方差分析检验两组 5 个时间点（即0\%，25\%，50\%，75\%，及100\%）FS，RPE及 各项生理指标指标变化的主效应及交互效应, 如主效应或 交互效应显著, 则采用Newman - Keuls法进行多重比较。 采用配对 T 检验判断两组运动后PAES量表得分之间的差 异性。统计学分析均使用SPSS 20.0软件 (IBM, 美国) 计算完成, 所有数据均以平均值士标准差 $($ Mean $\pm \mathrm{SD})$ 表示, 以 $P<0.05$ 为统计学差异显著, 以 $P<0.01$ 为统 计学差异非常显著。
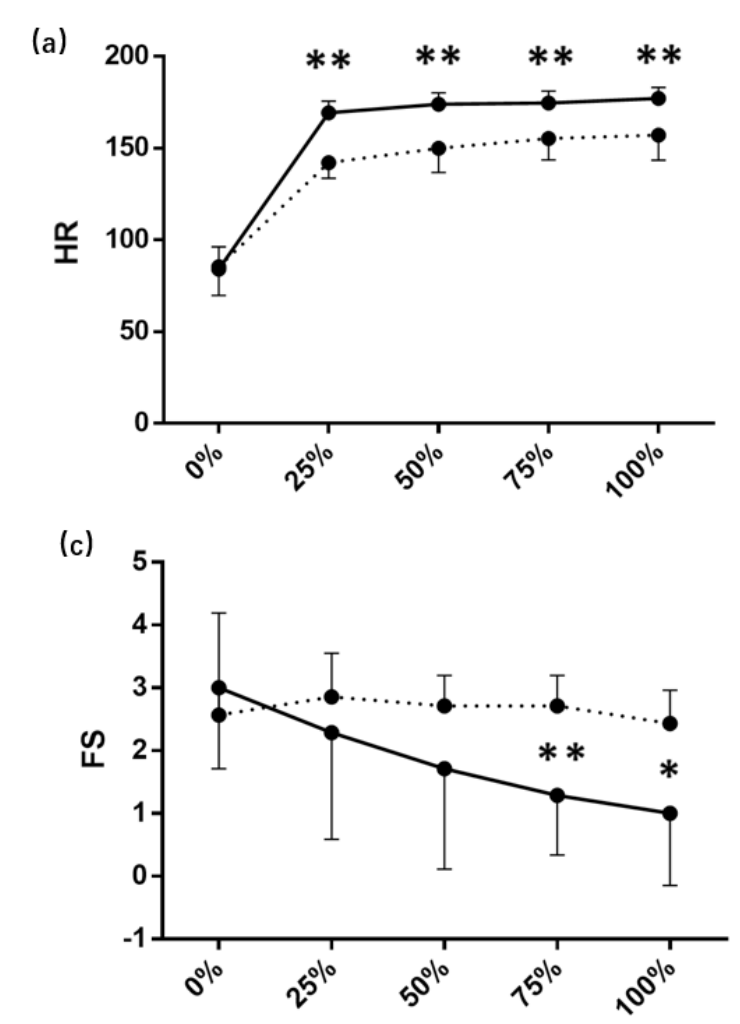

(b)

(d)
结果

两种运动时的HR、RPE、FS及LA比较

Figure 1所示, 对于四个指标, 受试者两次运动干 预前均无统计学差异。但两种运动对其影响均不同, 交 互效应存在 $(P<0.05)$ 。两次运动干预后各时间点, 受试者HR及RPE均高于运动前, 且统计学差异显著 $(\mathrm{P}<$ $0.01), H$ 组运动中HR及RPE各时间点均低于 $L$ 组, 且统 计学差异显著 $(P<0.01)$ 。两组在 $0 \% 、 25 \%$ 和 $50 \%$ 三个 时间点上FS值均无统计学差异。但在 $75 \%$ 及 $100 \%$ 点上, $H$ 组显著高于 $L$ 组 $(P<0.05)$ 。运动后两组的血乳酸浓度均 有显著提高（P<0.01），50\%及100\%点上H组的血乳酸浓 度均小于L组 $(P<0.01)$ 。

\section{两种运动的喜爱程度 (PAES) 对比}

Figure 2所示, 两组在运动后的PAES量表的得分上, $H$ 组要大于 $L$ 组且具有显著性差异, $P<0.05$ 。

Figure 1. (a) Representing changes in Heart Rate during exercise; (b) Representing changes in Ratings of Perceived Exertion during exercise; (c) Representing changes in Felling Scale during exercise; (d) Representing changes blood lactate in during exercise.

* Significantly different from corresponding $\mathrm{H}$ group value, $p<0.05$.
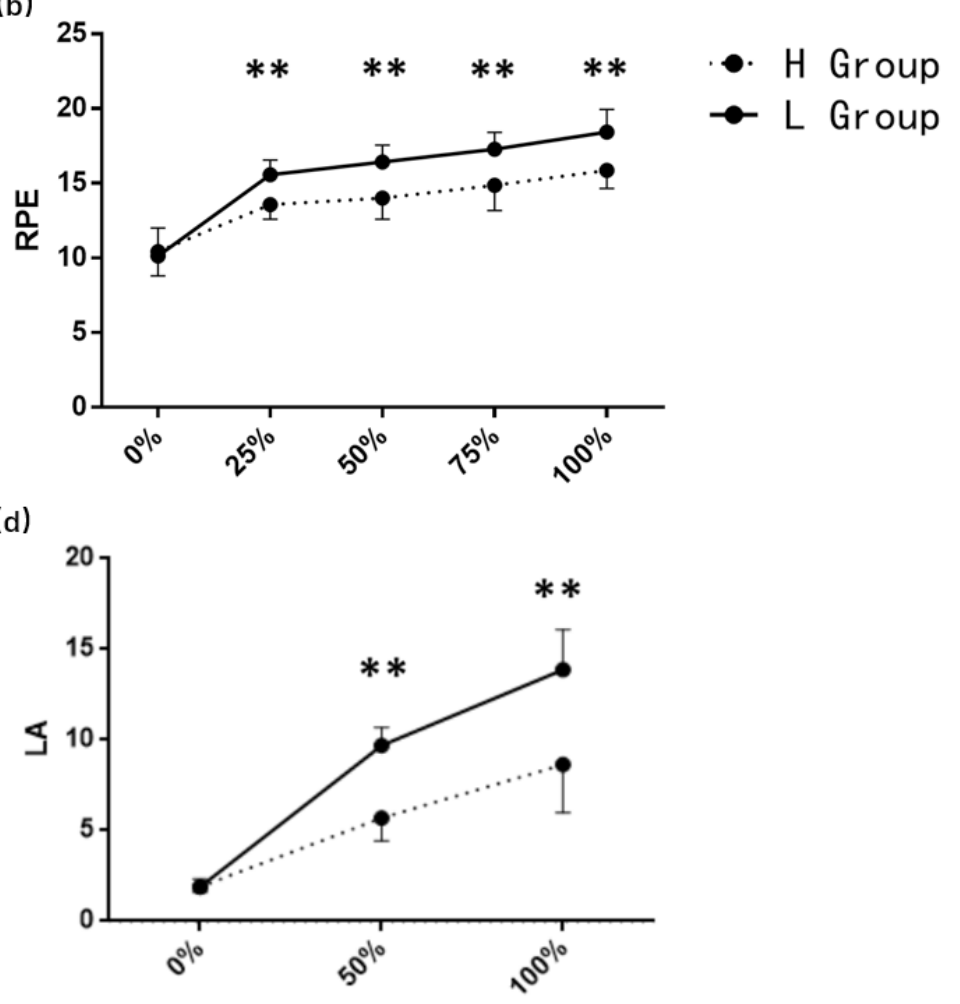

** Significantly different from corresponding $\mathrm{H}$ group value, $p<0.01$. 


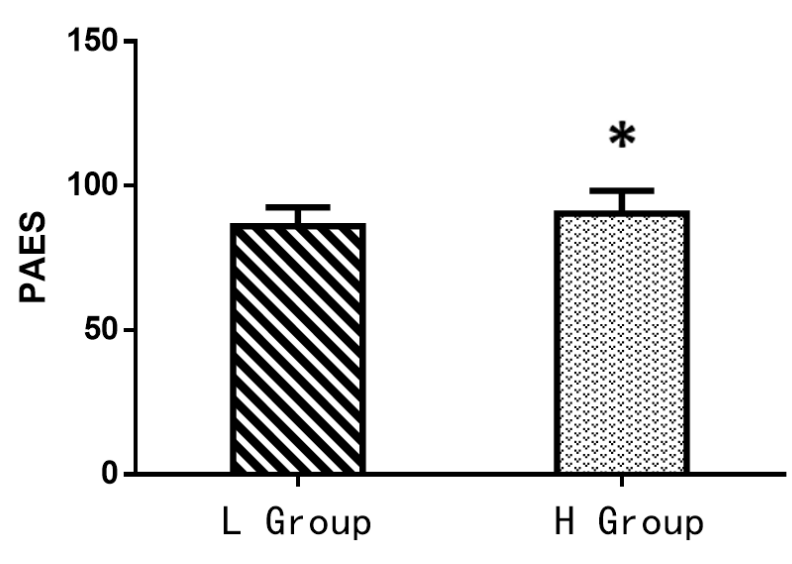

Figure 2. L Group represents the low-frequency high-intensity interval training group, and $\mathrm{H}$ group represents the high-frequency high-intensity interval training group.

* Significantly different from corresponding L group value, $p<0.05$.

\section{讨论}

本研究对比了高强度间歇运动时不同间歇频率对肥胖 青年女性运动愉悦性的影响。根据Buchheit等报导, HIIT 运动模式可以分为五类 [12], 本研究重点对其中两种间歇 形式进行对比，即低频间歇HIIT（运动时间 $>2-3 \min \left[13^{-}\right.$ 16]) 以及高频间歇HIIT (运动时间 $\geqslant 15 \mathrm{~s}[17-19]$ ) 。研 究结果发现H组与L组相比, 运动过程中FS量表得分和运动 后PAES量表得分更高, 同时发现其HR, LA及RPE较低。结 果提示, 同为HIIT, 高间歇频率短时间运动与低间歇频率 长时间运动相比, 运动的情感反应和愉悦性可能更好。其 原因可能是由于其运动时心率和血乳酸水平更低所导致。

最近HIIT的运动效果逐渐被人们所看好, 在其获得良 好减肥效果的同时也能够缩短运动时间, 然而运动所带 来的益处在停止训练后就会迅速消失。同时ACSM在其《 运动测试与处方指南 (第9版) 》中指出, 个人的喜好 有利于行为的出现与保持 $[20]$, 也就是说在运动中产生 的积极情感, 能使其坚持更长时间的运动计划 [21]。所 以, 通过增加运动方案中积极的情感反应 (affective response) 以及运动后的愉悦 (enjoyment) 来提高运 动依从性显得尤为重要。

有研究指出, 在MICT中随着运动时间的增加运动感觉 将会逐渐变坏 [22]。而不同间歇形式的HIIT由于其运动 形式的特殊性还并未发现有相关研究。根据Ekkekakis 提出的双模型理论 (The Dula-Mode Theory), 运动情 感是认知因素（包括自我效能, 目标或归因等) 和内感
因素 (呼吸, 心率或肌肉状态等) 在不同运动强度下交 互作用的结果, 其认为当运动强高于无氧阈时内感因素 为主导, 而内感因素主要与消极情感的变化有关 [23]。

所以在HIIT中, 内感因素的降低可能会导致运动中消 极情感减少。而HR, LA及RPE是反映内感因素的有效指 标。因此可以推断H组较L组有着更积极的情感反应可能 是由于H组所反映的HR、LA及RPE更低, 降低了其内感因 素。在Lukas Cipryan的研究中 [19], 通过对比 $100 \% \mathrm{VO}_{2 \text { peak }}$ 运动强度的两种HIIT ( 3 min $\times 3$ min VS $30 \mathrm{~s} \times 30 \mathrm{~s})$ 发现 低频间歇时平均HR及RPE均高于高频间歇, 其研究结果一 定程度上支持了本研究的结论。此外, HIIT间歇频率的降 低将会导致无氧酵解供能及神经肌肉负荷增加 [12], 而机 体的乳酸堆积同样会增加消极的运动情感 [7]。在Lukas Cipryan的研究中同时也对血乳酸情况进行了验证, 两种 间歇形式在运动中的血乳酸浓度出现了显著性差异, 低 频间歇的血乳酸值要显著高于高频间歇, 这与本研究出 现的结果相一致。但在Wesley J的研究中 [24], 使14名 男性受试者同样进行两种间歇形式的HIIT（4min $\times 4$ min VS $1 \mathrm{~min} \times 1 \mathrm{~min})$, 发现低频间歇组运动后的血乳酸浓 度虽然均值高于高频间歇组但无统计学差异 $(P=0.09)$ , 两项研究所产生不同结果可能是由于采取的受试人群 以及运动方案不同导致的。

分析认为两种运动形式产生血乳酸差异的原因可能是 由于：1）高频间歇HIIT拥有更加频繁的间歇期。并且 有研究表明 [25], 在正常运动条件下, 短暂的间歇期便 能使磷酸肌酸快速充分的恢复。所以, 多次的 $\mathrm{CP}$ 恢复可 能使高频间歇HIIT时ATP-CP系统供能频率增多, 从而一 定程度上降低了无氧供能时糖酵解的比例。2) 血乳酸 在运动时主要产生在骨骼肌中, 而骨骼肌中肌氧含量的 恢复和血乳酸的清除两者是相互的。肌氧恢复迅速, 组 织中氧含量高, 可以促进乳酸的氧化清除。同时乳酸的 清除可以改善机体的内环境, 有利于肌氧含量的恢复。 同时有研究指出, 肌氧含量恢复速率是呈曲线的形式, 在剧烈运动后前 $1 \mathrm{~min}$ 恢复较快后期逐渐减缓 [12]。而高 频间歇HIIT拥有更多的恢复间歇, 进而推测其可能更加 有利于肌氧含量的恢复, 从而促进氧化供能的进行。综 上两点原因, HIIT间歇频率的增加可能导致ATP-CP系统 供能比例的增加, 并且使肌肉中氧含量快速恢复, 在高 强度下运动时糖酵解供能比例减少, 从而导致机体乳酸 堆积的减少。

此外, 除了运动中的情感反应, 愉悦性的回顾评价也 
对运动的依从程度有着很大的影响 [20]。虽然两者概念 上有着相似点, 但运动情感更加偏向于运动时的基本反 应, 而运动后愉悦性的评估却主要受到认知, 成就及社 会因素等的影响 [26]。H组PAES得分较高可能是由于多 变的运动形式使受试者产生了较大的乐趣。研究表明, 间歇运动较持续运动来说有着更高的自我效能感, 对于 完成HIIT有着较大的信心。相比于持续运动, HIIT的间 歇性特征使得参与者能够获得更多连续的积极成就。具 体来讲, 由于HIIT连续的 “开-关” 性质, 使得受试者认 为能够在休息之后的大约 1 分钟内更加努力, 而在持续运 动则要求运动长达数十分钟。因此, HIIT将运动分解为短 暂可克服的剧烈运动, 可使受试者产生多次成功的经历, 这种 “开-关” 性质反过来可以有助于提高自我效能信念 [27］。而由于高频间歇HIIT构建的 “开-关” 单元更多, 所以推测其可能是通过增加 “开一关” 单元, 使受试者获 得更多的成就及自我效能感, 从而提高了运动的愉悦性。 总体来讲, 如果需要全面的评判一个运动方案的心理反 应就需要将运动中的情感反应与运动后的愉悦性评价进 行整体研究, 本研究在两个方面得出的结论较为一致。

但本研究局限性在于样本量较少且受试人群较单一, 本结论能否普及到全年龄段或其他健康水平的人群还需 以后加以充分验证。另外, 本研究未能将认知因素 (如 自我效能感, 目标, 归因等) 及内感因素 (肌肉疲劳水 平等) 进行全面测试, 仅能一定程度上对两项运动产生 的情感差异进行解释。

\section{Conflicts of Interest}

The authors have no conflicts of interest relevant to this article.

\section{References}

1. Trost SG, Owen N, Bauman AE, Sallis JF, Brown W. Correlates of adults' participation in physical activity: review and update. Med Sci Sports Exerc. 2002; 34(12):1996-2001.

2. Zhang H, Tong TK, Qiu W, et al. Comparable effects of high-intensity interval training and prolonged continuous exercise training on abdominal visceral fat reduction in obese young women. Journal of Diabetes
Research,2017,(2017-1-1). 2017; 2017(2):1-9.

3. Kong Z, Fan X, Sun S, Song L, Shi Q, Nie J. Comparison of high-intensity interval training and moderate-tovigorous continuous training for cardiometabolic health and exercise enjoyment in obese young women: a randomized controlled trial. PLoS One. 2016; 11(7): e0158589.

4. Smith AE, Eston R, Tempest GD, Norton B, Parfitt G. Patterning of physiological and affective responses in older active adults during a maximal graded exercise test and self-selected exercise. Eur J Appl Physiol. 2015; 115(9):1855-66.

5. Wittekind AL, Micklewright D, Beneke R. Teleoanticipation in all-out short-duration cycling. Br J Sports Med. 2011; 45(2):114-9.

6. Zheng Ziwei, Zhang Haifeng. Different intensity exercise for abdominal visceral fat advances in research on the effects of fat mass. Chinese Journal of Sports Medicine. 2018; 1: 70-77.

7. Hall EE, Ekkekakis P, Petruzzello SJ. The affective beneficence of vigorous exercise revisited. Br J Health Psychol. 2002; 7(Pt 1):47-66.

8. Astorino TA, Thum JS. Interval training elicits higher enjoyment versus moderate exercise in persons with spinal cord injury. J Spinal Cord Med. 2018; 41(1):7784.

9. Kilpatrick MW, Martinez N, Little JP, et al. Impact of highintensity interval duration on perceived exertion. Med Sci Sports Exerc. 2015; 47(5):1038-45.

10. Martinez N, Kilpatrick MW, Salomon K, Jung ME, Little JP. Affective and Enjoyment Responses to HighIntensity Interval Training in Overweight-to-Obese and Insufficiently Active Adults. J Sport Exerc Psychol. 2015; 37(2):138-49.

11. Kendzierski D, Decarlo KJ. Physical Activity Enjoyment Scale: Two validation studies. J Sport Exerc Psychol. 1991; 13(1):50-64.

12. Buchheit M, Laursen PB. High-intensity interval training, solutions to the programming puzzle. Part II: anaerobic energy, neuromuscular load and practical applications. Sports Med. 2013; 43(10):927-54. 
13. Pavey TG, Gartner CE, Coombes JS, Brown WJ. Assessing the effectiveness of High Intensity Interval Training (HIIT) for smoking cessation in women: HIIT to quit study protocol. BMC Public Health. 2015; 15:1309.

14. Isaksen K, Munk PS, Valborgland T, Larsen AI. Aerobic interval training in patients with heart failure and an implantable cardioverter defibrillator: a controlled study evaluating feasibility and effect. Eur J Prev Cardiol. 2015; 22(3):296-303.

15. Valstad SA, Heimburg EV, Welde B, Tillaar RVD. Comparison of Long and Short High-Intensity Interval Exercise Bouts on Running Performance, Physiological and Perceptual Responses. Sports Medicine International Open. 2018; 02(01):e20-e27.

16. Munk PS, Staal EM, Butt N, Isaksen K, Larsen AI. Highintensity interval training may reduce in-stent restenosis following percutaneous coronary intervention with stent implantation A randomized controlled trial evaluating the relationship to endothelial function and inflammation. Am Heart J. 2009; 158(5):734-41.

17. Maillard F, Rousset S, Pereira B, et al. High-intensity interval training reduces abdominal fat mass in postmenopausal women with type 2 diabetes. Diabetes Metab. 2016; 42(6):433-441.

18. Obradović J, Vukadinović M, Pantović M, Baić M. Hiit vs moderate intensity endurance training: impact on aerobic parameters in young adult men. Acta Kinesiologica. 2016.

19. Cipryan L, Tschakert G, Hofmann P. Acute and PostExercise Physiological Responses to High-Intensity Interval Training in Endurance and Sprint Athletes. J Sports Sci Med. 2017; 16(2):219-229.
20. Wankel LM. The importance of enjoyment to adherence and psychological benefits from physical activity. International Journal of Sport Psychology. 1993; 24(2):151-169.

21. Salmon J, Owen N, Crawford D, Bauman A, Sallis JF. Physical activity and sedentary behavior: a populationbased study of barriers, enjoyment, and preference. Health Psychology Official Journal of the Division of Health Psychology American Psychological Association. 2003; 22(2):178-88.

22. Thum JS, Parsons G, Whittle T, Astorino TA. HighIntensity Interval Training Elicits Higher Enjoyment than Moderate Intensity Continuous Exercise. PLoS One. 2017; 12(1):e0166299.

23. Ekkekakis P. Pleasure and displeasure from the body: Perspectives from exercise. Cogn Emot. 2003; 17(2):213-239.

24. Tucker WJ, Sawyer BJ, Jarrett CL, Bhammar DM, Gaesser GA. Physiological Responses to High-Intensity Interval Exercise Differing in Interval Duration. J Strength Cond Res. 2015; 29(12):3326-35.

25. Shen Youqing, Xu Guodong. Muscle oxygen content and blood lactate after increasing load exercise. Recovery research. China Sports Science and Technology. 2011; 05:73-77+85.

26. Roberts GC. Motivation in sport and exercise. Advances in Motivation in Sport \& Exercise. 1992; (1):193.

27. Jung ME, Bourne JE, Little JP. Where does HIT fit? An examination of the affective response to high-intensity intervals in comparison to continuous moderate- and continuous vigorous-intensity exercise in the exercise intensity-affect continuum. PLoS One. 2014; 9(12): e114541. 